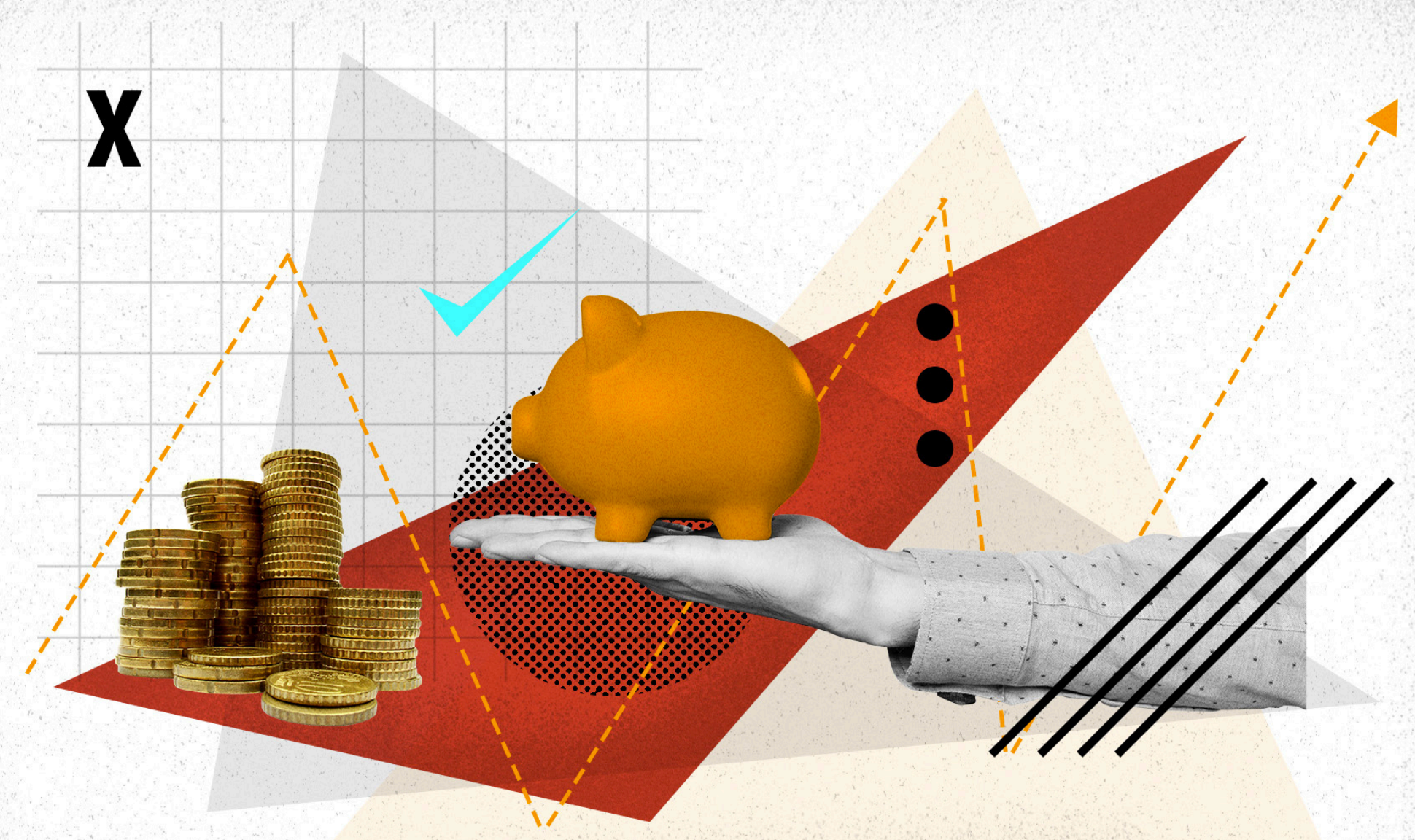

\title{
La educación financiera en primaria, una opción de trabajar matemáticas en el aula
}

FINANCIAL EDUCATION IN ELEMENTARY SCHOOL, AN OPTION TO WORK ON MATHEMATICS IN THE CLASSROOM

EDUCAÇÃO FINANCEIRA NO ENSINO FUNDAMENTAL, UMA OPÇÃO PARA TRABALHAR AS MATEMÁTICAS NA SALA DE AULA

Diana Maritza Vanegas García 


\section{Diana Maritza Vanegas García ${ }^{1}$}

Estudiante del Doctorado en Educación de la Universidad Federal Minas Gerais (Brasil); Magíster en Educación; Especialista en Educación Matemática, Licenciada en Pedagogía Infantil, Universidad Distrital Francisco José de Caldas. ORCID: http://orcid.org/0000-0002-57407894; correo electrónico: dmvanegasg@gmail.com

\section{Citar artículo como:}

Vanegas, D. (2020, Julio-Diciembre). La educación financiera en primaria, una opción de trabajar matemáticas en el aula. Revista Educación y Ciudad, No. 39, pp. 73-84. //doi.org/10.36737/01230425.n39.2020.2339

DOI: https://doi.org/10.36737/01230425.n39.2020.2339

Fecha de recepción: 1 de febrero de 2020 / Fecha de aprobación: 1 de julio de 2020

\section{Resumen}

Durante el año 2018 surgió el interés de trabajar la educación financiera con estudiantes de grado $5^{\circ}$ en una institución pública en la ciudad de Bogotá. Este artículo consolida la labor realizada, que giró alrededor de algunos objetivos, como buscar opciones pedagógicas que permitan proponer una enseñanza alternativa de las matemáticas; trabajar conceptos financieros, colocándolos en práctica y plantear problemas. Todo ello, se desarrolló en medio de un ambiente que permitió conocer a las familias, pensar en opciones para aprender sobre finanzas (ahorro, presupuesto, inversión) y también proyectarse a futuro (intereses profesionales).

Palabras clave: Educación financiera, intereses profesionales, ahorro, matemáticas en primaria, enseñanza de las matemáticas.

\section{Abstract}

During 2018, interest arose in working on financial education with 5th grade students from a public institution in Bogotá. This article consolidates the classroom work, looking for pedagogical options to propose an alternative teaching of mathematics, working on financial concepts and putting them into practice, while raising problems, knowing the families, thinking about options to learn about finance (savings, budget, investment) and also thinking about the future (professional interests).

Keywords: Financial education, professional interests, savings, mathematics in primary school, teaching of mathematics.

\section{Resumo}

Durante 2018, surgiu o interesse em trabalhar na educação financeira com estudantes da $5^{\mathrm{a}}$ série de uma instituição pública em Bogotá. Este artigo consolida o trabalho em sala de aula, buscando opções pedagógicas para propor um ensino alternativo de matemática, trabalhando conceitos financeiros e colocando-os em prática, levantando problemas, conhecendo as famílias, pensando em opções para aprender sobre finanças (poupança, orçamento, investimento) e também pensando no futuro (interesses profissionais).

Palavras-chave: educação financeira, interesses profissionais, poupança, matemática na escola primária, ensino das matemáticas. 


\section{Es necesario conocer nuestros estudiantes de otra manera}

Tos estudiantes tienen curiosidades, conocimi$\perp$ entos e intereses que muchas veces no son considerados en el día a día de la clase de matemáticas. El trabajo de aula habitual, que hemos visto por años como el método más adecuado: La transmisión repetitiva de conocimientos hasta llegar a la memorización; ha hecho que niños y niñas pierdan emoción al enfrentar algunas clases. Esto lleva a plantear distintas reflexiones sobre el quehacer pedagógico, es decir, a buscar alternativas de trabajo con los estudiantes; es en ese contexto que se ubica el proyecto que se expondrá a continuación, el cual involucra un trabajo con estudiantes de primaria.

El proyecto se desarrolló en la Institución Educativa Distrital Fernando Mazuera Villegas, de la localidad de Bosa, jornada tarde, con estudiantes de quinto grado durante el año 2018. Los objetivos giraron alrededor de una búsqueda por generar hábitos de ahorro y cuidado por medio de estrategias colectivas entre docente y estudiantes; con ello se buscó concretar el proyecto denominado "Educación matemática financiera en primaria”, en el cual se daba importancia a la generación de hábitos que pudiesen ser implementados por los estudiantes con sus familias, desde preguntas como ¿Quiénes somos? y ¿dónde estamos?; de tal forma que se facilitaran reflexiones sobre el reconocimiento de dónde venimos y hacia dónde queremos llegar, pensando en que ello puede transformar el camino presente para transitar un mejor futuro.
Desde hace algunos años, partiendo del propio lugar como docente investigadora, se fue cultivando la curiosidad por conocer la forma en que aprenden los sujetos, específicamente niños y niñas de primaria en el área de matemáticas; una reflexión que llevó a cuestionar las acciones que, como profesores, podemos hacer para que los niños se motiven en clase. De esta forma, junto a otros docentes, se logró consolidar un trabajo de pequeños pilotajes con proyectos llevados a cabo con estudiantes de colegios distritales y profesores en formación. Así, reconociendo que muchos de los proyectos realizados por docentes se quedan solo en el aula de clase, se da este siguiente paso: exponer ante la comunidad los avances obtenidos con esta labor concretada con estudiantes de $10 \mathrm{a}$ 12 años aproximadamente.

El trabajo partió de un diagnóstico, el cual incluyó algunas preguntas que ayudaron a conocer un poco las razones de los estudiantes para no cuidar su entorno escolar; entre ellas, se incluyeron preguntas como: ¿Por qué no cuido lo que me rodea en el colegio?; ¿he rayado (escrito) alguna pared, escritorio, mesa, silla o baño, etc.?; ¿por qué?; ¿qué escribo en estos lugares y por qué? Las preguntas diagnósticas se plantearon para que fuesen contestadas de forma anónima, con la intención de que los estudiantes escribieran sin sentirse acusados.

La falta de cuidado para con algunos recursos de la institución, condujo a indagar por alternativas pedagógicas que permitieran crear conciencia y entusiasmo; la educación financiera fue el espacio que facilitó una respuesta, generando unos tópicos o preguntas orientadoras que llevaron a plantear diferentes actividades, como: 1) Nada es gratis, 
todo tiene un costo; 2) ¿Realmente necesito todo?; 3) Intercambio, volviendo al trueque; 4) El presupuesto, realmente ¿cuánto puedo gastar?; 5) Consumidores astutos; y 6) Capacidad de endeudamiento.

\section{¿Por qué llegar a la educación matemática financiera?}

Luego de realizar distintas intervenciones en clase de matemáticas, como, por ejemplo: Utilizar competencias democráticas en las aulas con estudiantes de $1^{\circ}$ y $3^{\circ}$ de primaria (Mendoza y Vanegas, 2013); comprender que son sujetos sociales y participantes activos; o intentar que las matemáticas tengan relevancia y generen interés en ellos desde los proyectos de vida (Vanegas y Vanegas, 2014); se llegó a la conclusión de que este tipo de investigaciones permite encontrar relaciones, no solo con los contenidos matemáticos, sino con el entorno. Así, vale la pena destacar que, aunque el pensamiento aleatorio y el sistema de datos han permitido grandes avances, pues precisamente la estadística es la que permite a los estudiantes encontrar respuestas a varios interrogantes, es necesario seguir trabajando y pensar en otras posibilidades.

El presente artículo se basa en una revisión detallada de temas que pueden generar interés en los alumnos y aportar al ámbito cotidiano de sus familias; sin embargo, ¿cómo llevar a que existan iguales intereses en niños y niñas de primaria?; ¿cómo lograr que participen de manera activa en las decisiones de sus hogares?; ¿cómo lograr que valoren su entorno inmediato (salones, puestos, escritorios, libros, etc.)? Tales interrogantes se convirtieron en preguntas orientadoras que permitieron pensar un proyecto desde la clase de matemáticas. Así, luego de algunas revisiones y discusiones, se pensó en la posibilidad de trabajar desde la educación financiera, partiendo de algunas situaciones que pudieran ser tratadas en clase y reflexionadas en casa, partiendo de lo planteado por distintos autores, como Rubiano, quien, por ejemplo, afirma:

\begin{abstract}
Entre los beneficios que tiene el individuo cuando posee una educación financiera está el poder tomar decisiones financieras asertivas creando estrategias de ahorro e inversión correctas. Las personas con capacidades financieras podrán administrar sus cuentas y su patrimonio, lo cual se va a ver reflejado en una mejor calidad de vida para el individuo y la de su familia. Por otro lado, los beneficios para el país son notorios; el crecimiento de la economía de un país tiene en cuenta el ahorro. Cuando los individuos ahorran e invierten acertadamente, el crecimiento económico para la nación será mayor (Rubiano, 2013, p. 5).
\end{abstract}

De un tiempo para acá, la Educación Económica y Financiera (EEF) para todos se ha pensado como una necesidad. De hecho, entes internacionales como el Banco Mundial y la Organización para la Cooperación y el Desarrollo Económico (OCDE), han dado directrices para que sea trabajada en los diferentes países, incluyendo información en sus currículos; tal es el caso de Colombia, que cuenta con indicaciones específicas para trabajar la EEF.

Entidades como el Banco de la República o Asobancaria $^{2}$ han pensado en la EEF para trabajar con diferentes grupos poblacionales (Asobancaria, 2019), creando, por ejemplo, documentos como las orientaciones pedagógicas para la educación económica y financiera: Mi plan, mi vida y mi futuro, orientaciones pedagógicas para la educación económica y financiera (MEN, 2014) y la Estrategia Nacional de Educación Económica y Financiera de 
Colombia (ENEEF) (Comisión Intersectorial para la Educación Económica y Financiera (CIEEF), 2017). La preocupación por temas de la educación económica y financiera no solo se maneja en las facultades de ciencias económicas, ha llegado a entornos más sociales y cercanos a la gente, como periódicos, revistas y medios de comunicación que han destacado la importancia de enseñar finanzas desde muy temprana edad. Tales publicaciones permiten observar que existe una preocupación constante por brindar estrategias aplicables en el ámbito cotidiano del hogar, métodos y herramientas para tener una vida financiera más saludable. Sin embargo, esas directrices no son siempre conocidas por los docentes de aula regular, lo cual significa que pueden no estar presentes como herramientas de la escuela.

\section{¿Cómo desarrollar el trabajo?}

Desde hace algún tiempo, autores como Mendoza y Vanegas (2013) y Vanegas y Vanegas (2014) han realizado investigaciones alrededor del tema del interés de los estudiantes, implementando metodologías como los ambientes de aprendizaje (Skovsmose, 1999) y profundizado en aspectos como la modelación matemática, optando por desarrollar y estudiar ambientes de modelación partiendo de lo que Barbosa (2003 y 2006) ha llamado la perspectiva socio crítica; desde este punto de vista, crear y desarrollar ambientes de modelación implica dar prioridad al hecho de posibilitar reflexiones sobre el papel que desempeñan las matemáticas en la sociedad (Barbosa, 2006).

Gremio representativo del sector financiero colombiano, integrado por los bancos comerciales, nacionales y extranjeros, públicos y privados, además de las más significativas corporaciones financieras e instituciones oficiales especiales; al respecto es posible consultar: https://comparabien.com.co/sponsor/asobancaria
Junto a ello, partiendo de elementos propios de la Educación Matemática Crítica (EMC), entendiendo que las problemáticas abordadas pueden ser asumidas desde situaciones que a primera vista parecen no matemáticas, y que los temas de interés pueden ser elegidos por los estudiantes (Araújo, 2009) o planteados por los profesores para que funcionen como contexto, llevando a plantear interrogantes susceptibles de ser abordados y reflexionados (Barbosa, 2004; Camelo, Perilla y Mancera, 2016); fue posible justificar el proyecto de aula, reconociendo que la modelación matemática desde la EMC ha facilitado el desarrollo de la investigación, pues permite dar cuenta de una educación que aporta políticamente a los estudiantes del Colegio Fernando Mazuera Villegas.

Así, los siguientes principios de la EMC dan respuesta y sustentan la investigación que, como se ha dicho, se plantea desde la educación matemática financiera con estudiantes de quinto grado de primaria.

Lo social antecede a lo matemático: En las prácticas pedagógicas e investigativas bajo la perspectiva de la EMC, antes que definir qué contenidos matemáticos deben ser abordados, se debe establecer en qué situaciones los estudiantes están interesados, para abordarlas colectivamente y, a partir de allí, las matemáticas emergen gracias a procesos de modelación matemática [...] Las matemáticas no son neutrales. En nuestras prácticas cotidianas, las matemáticas no deben ser consideradas como un conocimiento neutral, pues son un conocimiento/ poder del cual individuos y colectividades, de 
manera consciente o inconsciente, hacen uso en diversas situaciones estimulando una determinada visión del mundo. Las prácticas de la educación matemática no pueden definirse exclusivamente en términos de procesos de pensamiento individual. Las dificultades, posibilidades y retos de las matemáticas escolares no se ubican "solamente en la "cabeza" de cada uno de los individuos, sino también en la manera como colectivamente y a través del contexto se construyen ideas sobre lo que es legítimo para la comunidad en que se está inmerso" (Camelo, Mancera y Salazar, 2017). Las prácticas en EMC requieren reconocer quienes son los participantes. En prácticas pedagógicas y de investigación en educación matemática es necesario e imperante indagar, utilizando diversos medios, por los actores involucrados en la creación y desarrollo de las situaciones a analizar (Camelo, Vanegas y Galvis, 2017).

Estas y otras razones de la práctica pedagógica han permitido consolidar la investigación desarrollada, la cual se sigue ejecutando en la institución educativa $\mathrm{y}$ ha encontrado avances significativos en los estudiantes, quienes, a partir de sus interrogantes, han logrado fortalecer sus estrategias de ahorro individual y familiar. De igual manera, el trabajo ha llevado a que los alumnos planteen aportes significativos al cuidado de lo que les rodea, pues son conscientes de las consecuencias de dañar lo público y entienden de dónde proviene lo que les rodea en la institución.

\section{¿Y en estos grados es urgente y necesario que los chicos sepan manejar sus finanzas?}

A diario, los estudiantes encuentran sillas dañadas y rayadas, no siempre cuentan con útiles escolares y enfrentan distintos inconvenientes comunes en algunas instituciones educativas; un diálogo directo permite evidenciar las difíciles situaciones familiares y carencias económicas y emocionales que les afligen, de las cuales han aprendido hasta incluso acostumbrarse. Como docente, tales situaciones afectan emocionalmente; no es fácil ver que estudiantes de quinto grado de primaria, quienes deben estudiar, llegar temprano para almorzar, ayudar en casa y responder con las tareas del colegio, deban enfrentar problemáticas familiares; sin embargo, siempre es posible notar su gran potencial, llamado "interés" y/o "curiosidad".

Desde allí, pero también desde algunos reflejos, como no cuidar las cosas de la institución, o su entorno personal y familiar, surge este proyecto, el cual inició con la intención de cuidar el entorno y, luego de algunas entrevistas y diálogos con los estudiantes, se propuso una enseñanza alternativa de las matemáticas, partiendo de que cada aula es un lugar de investigación, pensando en que la labor docente debe tener sentido, pues antes de los contenidos matemáticos, los niños deben estar preparados para manejar de manera asertiva diferentes situaciones sociales, entre ellas, sus finanzas, al punto de que puedan generar ahorro en sus hogares, no solo de dinero, sino de servicios (agua, luz, gas, etc.), creando conciencia social. 


\section{Educación financiera en primaria}

"Aprendo sobre educación financiera"

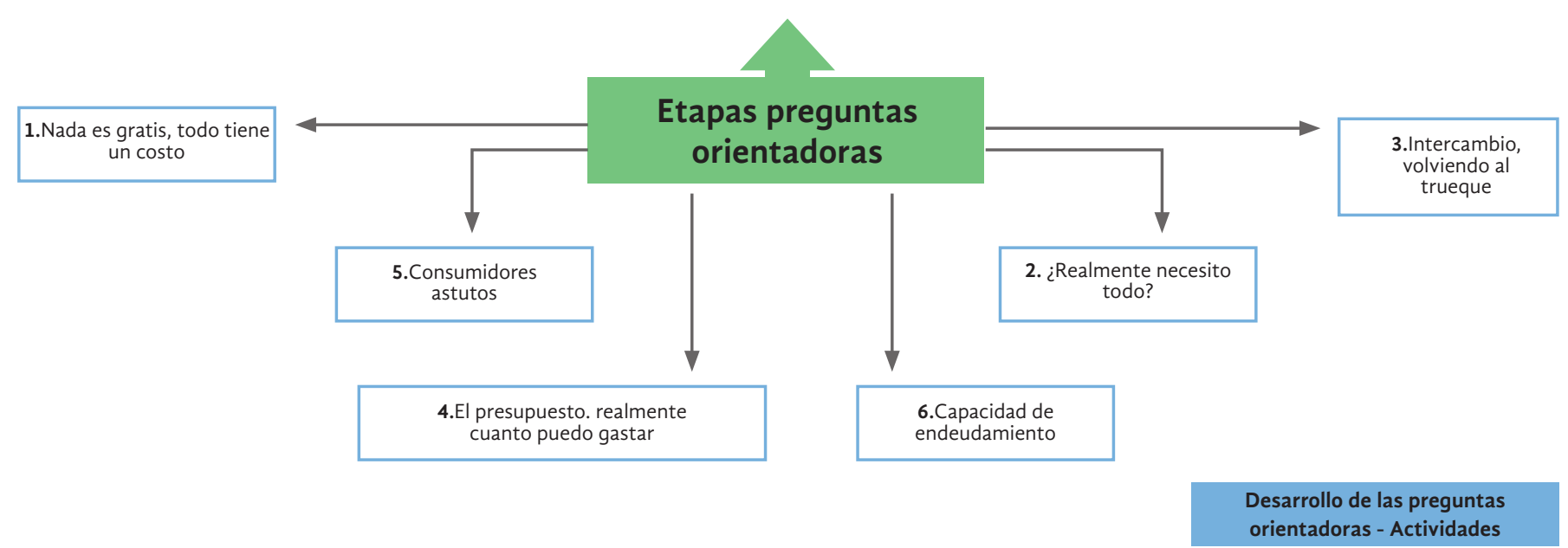

Nota. Fuente: Elaborado por autora

Las etapas o preguntas orientadoras, señaladas al principio del texto y que aparecen en la Figura 1, llevaron a organizar tablas como la presentada en la Figura 2 para cada una de las 6 etapas o preguntas orientadoras. La primera etapa fue "Nada es gratis, todo tiene un costo" y se dividió en 9 tópicos, anexando actividades como:

- Un collage que incorpora aquello que cada estudiante considera como de su propiedad; esto, con el objetivo de generar conciencia sobre nuestras propiedades (pues muchos de ellos pensaban que no tenían nada), para, de esta forma, aprender a valorar lo que hay en casa.

- A partir de la pregunta ¿Quiénes aportan en casa?, se buscó entender la economía familiar; los estudiantes hicieron un diagrama en el cuaderno, allí identificaban a los miembros de sus familias y las funciones de cada uno de ellos. La idea es que les reconozcan e identifiquen a quienes aportan económicamente en casa; sin embargo, el ejercicio también facilita reconocer a quienes cumplen con otras funciones que ayudan al desarrollo personal; de la misma forma, la actividad permitió retratar los tipos de familia.

- Por medio de entrevistas, se planteó una diferenciación entre profesiones y oficios; aquí fue de gran importancia reconocer los trabajos de los miembros de las familias y concienciar sobre las obligaciones, responsabilidades y demandas de cada uno para cada trabajo, pues, como bien sabemos, no se trata solo de ir a trabajar y recibir el sueldo. 
Figura 2. Tabla correspondiente a la primera etapa: "Nada es gratis, todo tiene un costo"

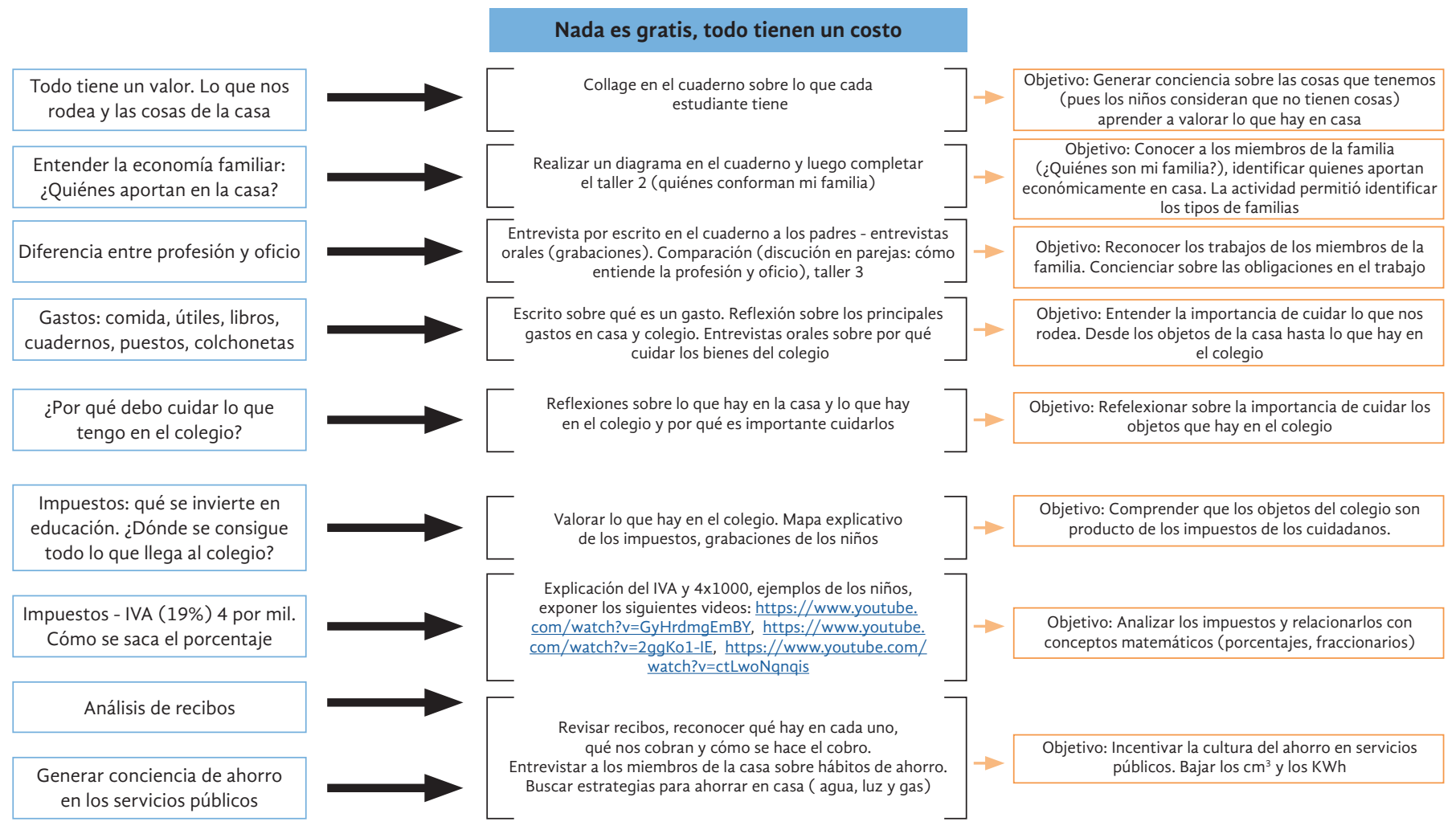

Por su parte, la reflexión sobre los ingresos disponibles de cada familia implicó clasificar los gastos (comida, útiles escolares, ropa, etc.), para entender la importancia de cuidar desde el reconocimiento del valor de lo que nos rodea, incluyendo los objetos de la casa y del colegio, para pensar en ¿por qué debo cuidar lo que tengo en el colegio? Todo, con el fin de generar interés y preocupación por los objetos del entorno, lo cual llevó a pensar en los impuestos, pues son la fuente que permite la llegada de recursos al colegio, intentando comprender que los distintos elementos no son algo regalado. Tales explicaciones llevan a analizar y entender el Impuesto del Valor Agregado
(IVA), el 4x1000 y los distintos porcentajes aplicables a diferentes objetos, hasta llegar al estudio de los impuestos y a establecer una relación con distintos conceptos matemáticos (porcentajes, fraccionarios, multiplicaciones, divisiones, entre otros).

Finalmente, esta primera pregunta orientadora llevó a realizar un análisis de los distintos recibos que llegan a casa (luz, agua, teléfono, internet, etc.), para reconocer los elementos que les componen y qué es lo que se cobra en cada uno de ellos, con la intención de incentivar la cultura del ahorro; el ejercicio también incluyó entrevistas y reflexiones 
sobre hábitos de ahorro en casa, para ayudar a bajar el consumo en los hogares.

Imagen 1. Trabajo en clase. Los recibos

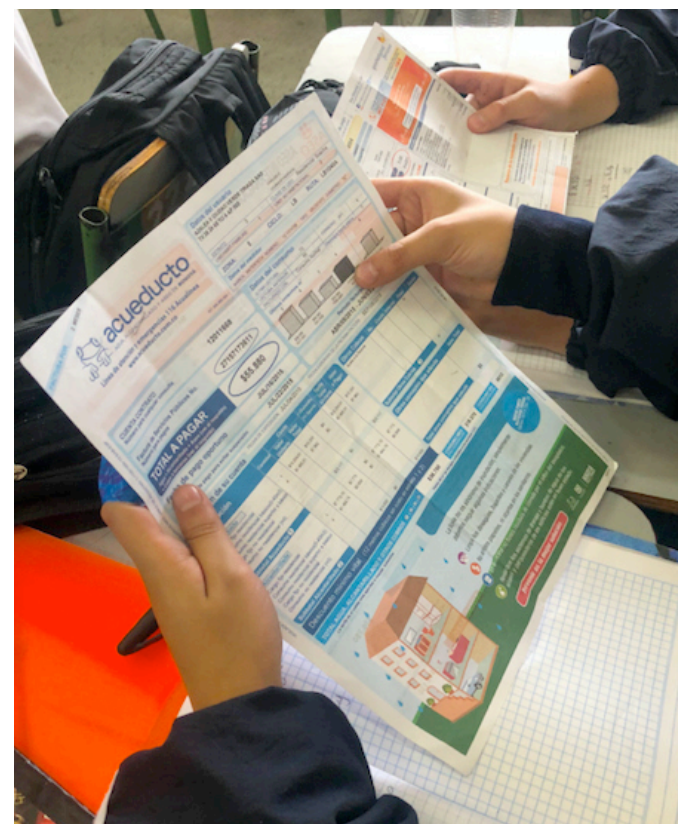

La segunda pregunta orientadora: “ ¿Realmente necesito todo?”; implicó un énfasis en el concepto de que "Nada es gratis, todo tiene un costo"; todo giró alrededor de un ejercicio que hizo clara la historia y la relación práctica de la vida con los billetes, no solo desde el reconocimiento de sus denominaciones, sino buscando entender sus componentes, quiénes son los personajes representados y los lugares emblemáticos que hacen parte de ellos; igualmente, se reconocieron las monedas de otros países y sus denominaciones (EE UU, Brasil, Canadá, Uruguay, Chile, México, Reino Unido, etc.), permitiendo a los estudiantes un diálogo sobre las diferentes monedas, mientras se planteaban cuestiones matemáticas (regla de tres, sumas, multiplicación); algunos llegaron a investigar aspectos relacionados con los países tratados.

Junto a ello, se planteó el objetivo de conocer y aprender el valor de los billetes y sus diferencias. Presentamos situaciones problema para que los estudiantes tomaran decisiones específicas, de tal forma que se les facilite entender los escenarios que enfrentan los padres en todo momento. En cuanto a las reflexiones sobre factores sociales, económicos y personales que influyen en la toma de decisiones, se asumió una estrategia que incluyó entrevistas a las familias, lo cual permitió llegar al punto de pensar en hábitos saludables (para tener en cuenta al momento de elegir un producto o alimento), generando un interés por el hecho de saber escoger las opciones adecuadas de acuerdo con cada situación, entendiendo también que algunas decisiones pueden ser temporales o definitivas.

Imagen 2. Trabajo en clase. Los billetes

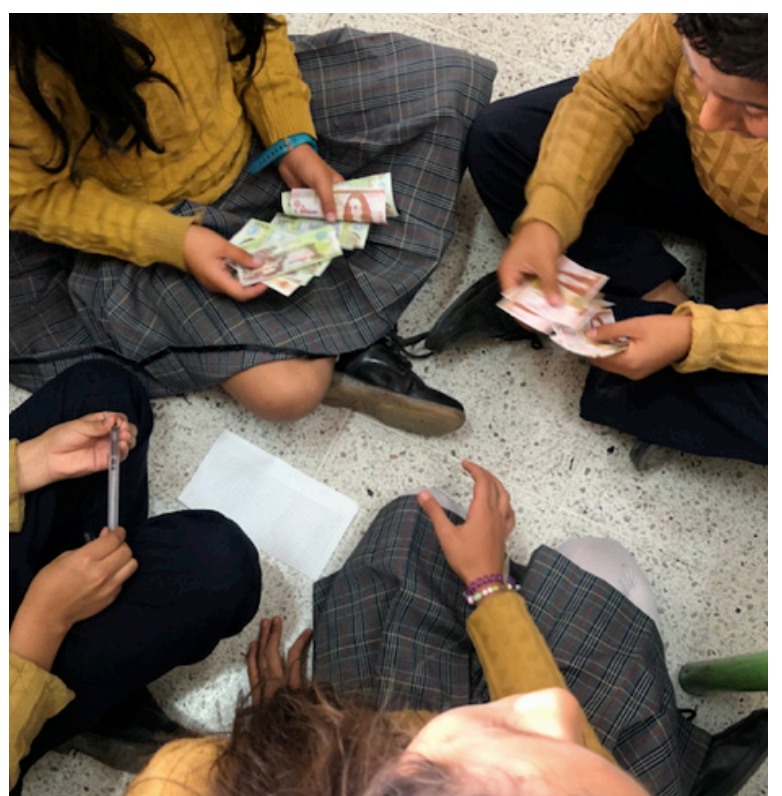


La tercera etapa, "Intercambio, volviendo al trueque", se dividió en tres subtemas que giraron alrededor de preguntas como: ¿Cuándo puedo intercambiar?; ¿qué se intercambia? El ejercicio consideró especialmente las cosas que se reciben en casa, como comida, ropa, etc., mientras el cambio consideraba diversas tareas, como realizar el aseo o colaborar de distintas formas; con ello se busca empezar a comprender qué es una responsabilidad. La actividad incorporó objetivos como valorar lo que nos rodea, cumplir, en consecuencia, con las obligaciones académicas y el valor de la responsabilidad, no solo para el colegio, sino en casa.

La cuarta pregunta orientadora, ¿Realmente, cuánto puedo gastar?, se planteó a partir de 6 puntos específicos; uno de ellos fue entender el concepto de salario mínimo (descuentos), e incluyó ejercicios de comparar el salario nacional con el de otros países, comprender las facturas, analizando aquellas disponibles en casa, que incluyeron recibos de comida, compras, etc. Esto no solo permitió desarrollar el tema, sino resolver dudas vinculadas al apartado del IVA. La idea es dialogar sobre los gastos presentes en las familias que devengan un salario mínimo y reflexionar sobre el concepto del ahorro.

La quinta fase, denominada "Consumidores astutos. Lo que realmente necesitamos", se relacionó con la cuarta pregunta, pues se plantean los gastos generales para entender la economía de una familia que dispone de un salario mínimo para solventar sus necesidades; el trabajo incluyó la identificación de las consignaciones y un diálogo sobre la diferencia entre cuenta de ahorro, cuenta corriente, tarjetas de crédito y de debito, diligenciando consignaciones y haciendo énfasis en la importancia del ahorro; por supuesto, se emplearon diferentes operaciones matemáticas para justificar cada una de las transacciones.

Finalmente, para el apartado de "Capacidad de endeudamiento e inversiones", se trataron temas como los gastos y los intereses generados por los créditos (de vivienda, vehículos, consumo, etc.), para destacar la importancia de realizar compras saludables, pensando en las diferentes situaciones (prioridades) y en la necesidad de ver más allá del día a día. Esta etapa dio comienzo a la discusión sobre el proyecto de vida, a partir de la pregunta: ¿Qué quiero ser cuando sea grande? Todo, buscando conocer los sueños de los niños y niñas, mientras se proponían mecanismos que incentivaran la búsqueda de alternativas a futuro, por medio de la conciencia del ahorro.

Los estudiantes se entusiasmaron, hicieron investigaciones para conocer universidades con la idea de proyectarse a futuro y pensar en un ahorro responsable. Para cerrar, se hizo entrega de una alcancía personalizada a cada estudiante delante de sus padres, de tal forma que, como familia, pensaran en un futuro mutuo, resaltando el porvenir de los niños y niñas de quinto.

Imagen 3. Alcancías personalizadas pensando en el futuro
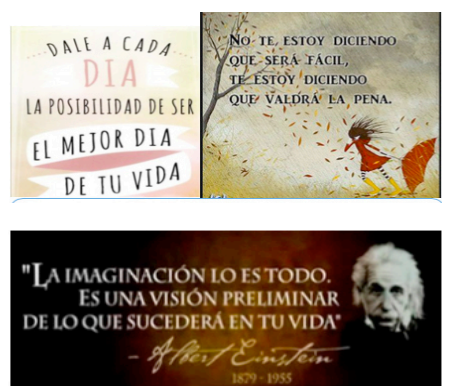


\section{¿Aparecieron los contenidos matemáticos que tanto nos preocupan?}

Una de las preocupaciones permanentes del trabajo fue dar respuesta al plan de estudios de la institución y a las exigencias externas; sin embargo, este tipo de ejercicio, que permite el diálogo continuo entre pares y maestros, lleva a desprenderse de esas inquietudes, con la ventaja de que las matemáticas son protagonistas de todo el proceso.

Por logeneral, los docentes de un país como Colombia parten de un currículo que propone las clases como punto de partida para la construcción colectiva del conocimiento, la incorporación de aspectos culturales y la aplicación del contenido matemático en la vida cotidiana (MEN, 1995, p. 54); por ello, el desarrollo de este tipo trabajo implica comprender situaciones que inquieten a los estudiantes, mientras permiten abordar de manera sencilla la realidad que les rodea, generando conocimiento colectivo. Dichas estrategias de enseñanza deben partir del trabajo en grupo, pensando en una nueva opción: incluir temas que puedan aportar al ámbito cotidiano y un enfoque interdisciplinar; tal es el caso específico de este trabajo de aula, pues, como señalara Freire:

La práctica docente crítica, que implica el pensamiento correcto, envuelve el movimiento dinámico, dialéctico, entre el hacer y el pensar sobre el hacer [...] por eso es que, en la formación permanente de los profesores, el momento fundamental es el de la reflexión crítica sobre la práctica. Es pesando críticamente la práctica de ayer y de hoy que se puede mejorar la próxima práctica (Freire, 2019, p. 39).
Las contribuciones a la reflexión crítica sobre la práctica deben partir de pensar conjuntamente en los estudiantes-docentes. 


\section{Referencias}

Araújo, J. (2009). Uma abordagem sócio-crítica da modelagem matemática: a perspectiva da educação matemática crítica. ALEXANDRIA, Revista de Educação em Ciência e Tecnologia, Vol. 2, No. 2, pp. 55-68.

Asobancaria. (2019). Compara bien. Obtenido el 13 de septiembre de 2019 desde https://comparabien. com.co/sponsor/asobancaria

Barbosa, J. (2003). Modelagem matemática e a perspectiva sócio-crítica. II Seminário Internacional de Pesquisa em Educação Matemática, Vol. 2, pp. 1-13.

Barbosa, J. (2004). Modelagem matemática: o que é? Por que? Como? Veritati, No. 4, pp. 73-80.

Barbosa, J. (2006). Mathematical modelling in classroom: A socio-critical and discursive perspective. ZDM, 38(3), PP. 293-301. DOI: https:// doi.org/10.1007/BF02652812

Camelo, F., Perilla, W., y Mancera, G. (2016). Prácticas de modelación matemática desde una perspectiva socio crítica con estudiantes de grado undécimo. Revista Latinoamericana de Etnomatemática, 9(2), pp. 67-84. DOI: https://doi.org/10.22267/ relatem. 1692.3

Camelo, F., Vanegas, D., y Galvis, T. (2017). Alzas en las tarifas de transporte público en Bogotá: un pretexto para explorar ambientes de modelación matemática. Trabajo presentado en la X conferencia Nacional sobre modelación en educación matemática. Maringa, Brasil.
Comisión Intersectorial para la Educación Económica y Financiera (CIEEF). (2017). Estrategia Nacional de Educación Económica y Financiera de Colombia (ENEEF). Bogotá: CIEEF.

Freire, P. (2019). Pedagogia da autonomía. Saberes necessários à prática educativa. Río de Janeiro/São Paulo: Paz \& Terra.

Mendoza, A., y Vanegas, D. (2013). Desarrollo de competencias democráticas a través de la educación estadística crítica con estudiantes de $1^{\circ}$ y $3^{\circ}$ grado. Tesis de especialización no publicada. Especialización en Educación Matemática. Bogotá, Colombia.

Ministerio de Educación Nacional de Colombia (MEN). (1995). Ley General de educación. Bogotá: Universidad Nacional de Colombia.

Ministerio de Educación Nacional (MEN). (2014). Mi plan, mi vida y mi futuro, orientaciones pedagógicas para la educación económica y financiera. Documento $N^{o} 26$. Bogotá: MEN.

Rubiano, M. (2013). Educación financiera en Colombia. Tesis en Economía y finanzas internacionales, no publicada. Bogotá, Universidad de la Sabana.

Skovsmose, O. (1999). Hacia una filosofía de la educación matemática crítica. Bogotá: Una empresa docente, Universidad de los Andes.

Vanegas, D., y Vanegas, C. (2014). La clase de matemáticas y la construcción del proyecto de vida. Estudiantes de grado undécimo del colegio Francisco Javier Matiz. Tesis de Maestría no publicada, Bogotá, Universidad Distrital Francisco José de Caldas. 